\title{
Distinct Patterns in Human Milk Microbiota and Fatty Acid Profiles Across Specific Geographic Locations
}

\section{OPEN ACCESS}

Edited by:

Mike Taylor,

University of Auckland, New Zealand

Reviewed by:

Jan S. Suchodolski,

Texas A\&M University, USA

Catherine Maree Burke,

University of Technology Sydney,

Australia

*Correspondence:

Himanshu Kumar kumhim@utu.fi

Specialty section:

This article was submitted to

Microbial Symbioses,

a section of the journal

Frontiers in Microbiology

Received: 30 June 2016 Accepted: 28 September 2016 Published: 13 October 2016

Citation:

Kumar H, du Toit E, Kulkarni A, Aakko J, Linderborg KM, Zhang $Y$, Nicol MP, Isolauri E, Yang $B$ Collado MC and Salminen S (2016)

Distinct Patterns in Human Milk Microbiota and Fatty Acid Profiles Across Specific Geographic Locations. Front. Microbiol. 7:1619. doi: 10.3389/fmicb.2016.01619

\begin{abstract}
Himanshu Kumar' ${ }^{*}$, Elloise du Toit' ${ }^{2}$ Amruta Kulkarni ${ }^{3}$, Juhani Aakko ${ }^{1,3}$, Kaisa M. Linderborg ${ }^{3}$, Yumei Zhang ${ }^{4}$, Mark P. Nicol5,6, Erika Isolauri ${ }^{7}$, Baoru Yang ${ }^{3}$, Maria C. Collado ${ }^{1,8}$ and Seppo Salminen ${ }^{1}$

${ }^{1}$ Functional Foods Forum, Faculty of Medicine, University of Turku, Turku, Finland, ${ }^{2}$ Division of Medical Microbiology, Department of Pathology, University of Cape Town, Cape Town, South Africa, ${ }^{3}$ Food Chemistry and Food Development, Department of Biochemistry, University of Turku, Turku, Finland, ${ }^{4}$ Department of Nutrition and Food Hygiene, School of Public Health, Peking University, Beijing, China, ${ }^{5}$ Institute of Infectious Disease and Molecular Medicine, University of Cape Town, Cape Town, South Africa, ${ }^{6}$ National Health Laboratory Service of South Africa, Groote Schuur Hospital, Cape Town, South Africa, ${ }^{7}$ Department of Pediatrics, University of Turku, Turku, Finland, ${ }^{8}$ Institute of Agrochemistry and Food Technology, National Research Council (IATA-CSIC), Valencia, Spain
\end{abstract}

Breast feeding results in long term health benefits in the prevention of communicable and non-communicable diseases at both individual and population levels. Geographical location directly impacts the composition of breast milk including microbiota and lipids. The aim of this study was to investigate the influence of geographical location, i.e., Europe (Spain and Finland), Africa (South Africa), and Asia (China), on breast milk microbiota and lipid composition in samples obtained from healthy mothers after the 1 month of lactation. Altogether, 80 women (20 from each country) participated in the study, with equal number of women who delivered by vaginal or cesarean section from each country. Lipid composition particularly that of polyunsaturated fatty acids differed between the countries, with the highest amount of n-6 PUFA (25.6\%) observed in the milk of Chinese women. Milk microbiota composition also differed significantly between the countries $(p=0.002)$. Among vaginally delivered women, Spanish women had highest amount of Bacteroidetes (mean relative abundance of 3.75 ) whereas Chinese women had highest amount of Actinobacteria (mean relative abundance 5.7). Women who had had a cesarean section had higher amount of Proteobacteria as observed in the milk of the Spanish and South African women. Interestingly, the Spanish and South African women had significantly higher bacterial genes mapped to lipid, amino acid and carbohydrate metabolism $(\rho<0.05)$. Association of the lipid profile with the microbiota revealed that monounsaturated fatty acids (MUFA) were negatively associated with Proteobacteria $(r=-0.43, p<0.05)$, while Lactobacillus genus was associated with MUFA ( $r=-0.23, p=0.04)$. These findings reveal that the milk microbiota and lipid composition exhibit differences based on geographical locations in addition to the differences observed due to the mode of delivery.

Keywords: microbiome, human milk, fatty acids, geography, delivery 


\section{INTRODUCTION}

Breastfeeding has an influential role in the increasing burden of non-communicable disease, including allergy and obesity (Aaltonen et al., 2011; Kelishadi and Farajian, 2014). Not only does it provide complete personalized nutrition to infants and is important for optimal growth and development, but it also confers immunological protection during critical period in life. A delicate balance of stimulatory, even inflammatory, maturational signals, together with myriad of anti-inflammatory compounds is transferred from mothers to infant via breastfeeding. The most potent immunoregulatory factors include cytokines, growth factors, specific proteins such as lactoferrin, peptides, fatty acids, human milk oligosaccharides, and microbes (Collado et al., 2012).

Breastfeeding mothers, however, do not comprise a uniform group. Maternal environmental conditions such as dietary habits and lifestyle, individual and circadian variations in fatty acid synthesis, genetic factors, and lactation time, all influence the composition of breast milk (Boersma et al., 1991; Brenna et al., 2007; Mäkelä et al., 2013). Recent reports have suggested antibiotics, maternal health and gestational age as factors impacting milk microbiota (Hunt et al., 2011; Cabrera-Rubio et al., 2012, 2016; Khodayar-Pardo et al., 2014; Soto et al., 2014; Hoashi et al., 2015). Intriguingly, mode of delivery is also known to affect breast milk microbiota composition (Collado et al., 2012; Khodayar-Pardo et al., 2014; Cabrera-Rubio et al., 2016).

Since the composition of breast milk shows marked individual variation, this may affect the immunoregulatory properties of breast milk. However, population or region-specific factors influencing microbiota composition are largely unexplored. Similarly, the potential relationship of milk microbiota with other milk compounds such as lipids has not been well established.

As personalized breast feeding provides the exclusive nutrition to an infant guiding the development of the gut microbiota and maturation of the immune system, we aimed to identify the impact of four different geographical locations: Asia, Africa, and North and South Europe on breast milk composition. We focused on the microbiome and the fatty acid composition and the impact of mode of delivery on breast milk composition across those locations.

\section{MATERIALS AND METHODS}

\section{Breast Milk Sample Collection}

The study group comprised 80 healthy women volunteers from different geographical locations representing urban lifestyles including China (Beijing area), South Africa (Cape Town), Finland (southwestern area), and Spain (Valencia, Mediterranean area). Subjects from each country $(n=20)$ were grouped according to the mode of delivery, vaginal $(n=10)$ and cesarean section $(n=10)$.

Maternal characteristics such as age, weight, body mass index (BMI) and parity were collected at the time of enrolment. All participants received detailed information about the study, written informed consent was obtained and the study protocol was approved by the Ethics Committees of the respective participating institutions, Spain (Bioethics Committee of CSIC and from the Regional Ethics Committee for Biomedical Research), Finland (Turku University Hospital), China (Medical Research Board of Peking University), and South Africa (University of Cape Town, Human Research Ethics Committee).

Before sample collection, the mothers were given oral and written instructions for standardized collection of samples. The mature milk samples (1 month post-partum) were collected manually into a sterile tube. Prior to collection, nipples and mammary areola were cleaned with soap and sterile water and soaked with chlorhexidine to reduce contamination by skin flora. The first drops of milk (approximately $500 \mu \mathrm{L}$ ) were discarded. All the samples were kept frozen at $-20^{\circ} \mathrm{C}$ until delivery to the laboratory and then stored at $-80^{\circ} \mathrm{C}$ until further analysis. All the samples were shipped to Finland for storage, processing, lipid analysis ,and DNA extraction as a part of collaborative project.

\section{Extraction of Lipids and Isolation of Triacylglycerols (TAGs) and Phospholipids (PLs)}

An internal standard mixture of triheptadecanoin (SigmaAldrich, St.Louis, MO, USA) and dinonadecanoylphosphatidylcholine (Sigma-Aldrich, St.Louis, MO, USA) was added to the thawed milk (from 178 to $539 \mathrm{mg}$ ). Then $1.5 \mathrm{~mL}$ methanol, $3 \mathrm{~mL}$ chloroform and $0.8 \mathrm{~mL} 0.88 \% \mathrm{KCl}$ in water were added and the blend was thoroughly vortexed. The tubes were centrifuged $2000 \times g$ for $3 \mathrm{~min}$ to separate the layers, and the chloroform rich layer was collected, evaporated to dryness, and re-suspended in chloroform (Folch et al., 1957). The triacylglycerols (TAG) and phospholipids were isolated from the extracted lipid mixture with solid phase extraction based on silica columns as described previously (Hamilton and Comai, 1988).

\section{Preparation of Fatty Acid Methyl Esters and Their Chromatographic Analysis}

Fatty acid methyl esters (FAME) were prepared with the sodium methoxide method (Christie, 1982; Hamilton and Comai, 1988). In short, the lipids were suspended in $1 \mathrm{~mL}$ dry diethylether; then $25 \mu \mathrm{L}$ methylacetate and $25 \mu \mathrm{L}$ sodium methoxide were added, and the blend was incubated for $5 \mathrm{~min}$ with shaking. The reaction was stopped with $6 \mu \mathrm{L}$ of acetic acid. The tubes were centrifuged $2000 \times g$ for $5 \mathrm{~min}$, after which the supernatant was collected and gently evaporated to dryness, and the resulting FAME were dissolved in hexane. The FAME were analyzed with gas chromatography (Shimadzu GC-2010 equipped with AOC-20i auto injector, flame ionization detector, Shimadzu corporation, Kyoto, Japan). A wall-coated open tubular column DB-23 $(60 \mathrm{~m} \times 0.25 \mathrm{~mm}$ i.d., liquid film thickness $0.25 \mu \mathrm{m}$, Agilent technologies, J.W. Scientific, Santa Clara, CA, USA) was used for the analysis. Helium was used as the carrier gas. Splitless/split injection was used, and the split was opened after $1 \mathrm{~min}$. The injection volume was $0.5 \mu \mathrm{L}$, and inlet temperature $270^{\circ} \mathrm{C}$. The initial oven temperature, which was held for $1 \mathrm{~min}$ was $130^{\circ} \mathrm{C}$. The oven temperature was programmed to rise at a rate of $4.5^{\circ} \mathrm{C} / \mathrm{min}$ to $170^{\circ} \mathrm{C}$ and $10^{\circ} \mathrm{C} / \mathrm{min}$ to $220^{\circ} \mathrm{C}$, where 
it was held for $3.5 \mathrm{~min}$, and further at $10^{\circ} \mathrm{C} / \mathrm{min}$ to $230^{\circ} \mathrm{C}$ and $60^{\circ} \mathrm{C} / \mathrm{min}$ to $240^{\circ} \mathrm{C}$, where it was held for $7 \mathrm{~min}$. The detector temperature was $280^{\circ} \mathrm{C}$. Peaks were identified by comparison of their retention times to the retention times of known external standard mixtures, Supelco 37 Component FAME Mix (Supelco, St. Louis, MO, USA), 68D (Nu-Check-Prep, Elysian, MN, USA), GLC-11A (NuCheck Prep, Elysian, MN, USA), and GLC-490 (Nu-Check-Prep, Elysian, MN, USA), and quantified in relation to the internal standards and corrected with response factors calculated based on analysis of standard mixtures. The identified fatty acids, i.e., TAG and PLs, were (grouped for further analysis) classified as saturated fatty acids (SAFA), monounsaturated fatty acids (MUFA) and polyunsaturated fatty acids (PUFA), and PUFA further sub-classified into n-3 and n-6 PUFAs.

\section{Microbial DNA Extraction and Sequencing}

Breast milk samples were centrifuged at $14,000 \mathrm{rpm}$ for $20 \mathrm{~min}$ at $4^{\circ} \mathrm{C}$, fat was removed and the pellet was used for total DNA extraction. Bead beating was carried out using FastPrep $^{\circledR}$ (FP120-230, Bio 101 ThermoSavant, Holbrook, NY), and DNA extracted from the supernatant using the InviMag ${ }^{\circledR}$ Stool DNA kit (Stratec Molecular, Berlin, Germany) with the KingFisher magnetic particle processor (Thermo Fisher Scientific Oy, Vantaa, Finland). Purified total genomic DNA was shipped to South Africa for PCR and sequencing. PCR amplification and sequencing was carried out at the Centre for Proteomic and Genomic Research (Cape Town, South Africa) using Illumina MiSeq sequencing platform. Primers targeting hypervariable V4 region of the bacterial 16S rRNA gene were used (bacterial/archaea primers $515 \mathrm{~F}$ and 806R) according to previously described methods and modified for the Illumina MiSeq platform. Controls included two no template controls (NTC) and two NTC spiked with 6 different Staphylococcus species at $20 \mathrm{ng} / \mathrm{uL}$ each. Sequencing data has been submitted to NCBI with SRA accession: SRP082263 and submission ID: SUB1772296.

\section{Microbial Community Analysis}

Quality assessment of obtained reads was done using prinseq-lite program (Schmieder and Edwards, 2011) with defined parameters (i.e., min_length:50, trim_qual_right:20, trim_qual_type:mean, trim_qual_window:20). Filtered and demultiplexed sequences were processed using the open-source software QIIME (with default parameters; Caporaso et al., 2010, 2012). A total of 80 samples were sequenced, with mean of 11,538 sequences per sample (500 minimum sequences per sample). One of the Finnish samples generated fewer than 500 sequences after quality filtering and was excluded from further analysis. The remaining sequences were then binned into Operational Taxonomic Units (OTUs) using de novo OTU picking based on $97 \%$ identity using the August 2013 build from Greengenes reference database. Alpha diversity was determined from rarefied tables using indices which includes Shannon-Wiener index, Chao 1 index for richness and Observed Species (number of unique OTUs) and Phylogenetic Distance (PD_whole) were also determined. Beta diversity was computed with OTU table using UniFrac. Weighted and unweighted unifrac distance along with sample metadata was used as input for principal coordinate analysis (PCoA). OTU table was rarefied to 500 sequences per sample for all samples, in computing diversity measures to avoid variations in sequencing depth. Calypso version $5.2^{1}$ was used with data transformed by centered log ratio with total sum normalization, to generate Venn diagram for shared phylotypes at family level, and Redundancy Analysis (RDA) plot for multivariate analysis using OTU phylotypes, and country as factor.

\section{Metagenomic Prediction}

Functional metagenomes were predicted from 16S rRNA reads using PICRUST (Langille et al., 2013). Briefly, OTU's based on the closed reference OTU picking method were selected using QIIME v 1.9.1 and by querying the data against a reference database (GreenGenes database v 13_8); OTUs were assigned at $97 \%$ identity. The resulting OTU table was used to predict metagenomes, which were functionally categorized based on KEGG (Kyoto Encyclopedia of Genes and Genomes) pathways at different levels of classification. The data was analysed using galaxy interface ${ }^{2}$ for LEfSe [Linear Discriminant Analysis (LDA) Effect Size] to elicit differential microbial functional pathways in different samples. The differential abundant features are ranked by effect size after LDA with effect size threshold between 2 and 3 (on a $\log _{10}$ scale).

\section{Statistical Analysis}

Relative abundance of fatty acids in TAG and phospholipids were statistically tested using two-factor analysis of variance followed by Tukey's HSD post hoc test to compare the main effects of country of origin and mode of delivery; and the interaction effect between country of origin and mode of delivery. Microbial group significance based on phylotypes was compared at different levels by ANOVA based on the QIIME pipeline. Pearson's correlation matrix was calculated for the gut microbiota (phylum and genus level) and the TAG and phospholipids. Heatmaps of correlation coefficients and principal component analysis were constructed using the g-plots and factoextra package in R ( R Core Team, 2010). The statistical analysis was performed with $\mathrm{R}$ statistical environment version 3.1.1.

\section{RESULTS}

\section{Subject Selection}

The mean age of the women from the four countries $(n=80)$ varied from 32 to 34, with mean pre-gestational BMI of 2425 (normal weight), except the Chinese group with mean BMI of 21.7 (SD \pm 1.9 ) (normal weight, because of Asian origin) $(p<0.05)$. All women recruited in the study, intended to breastfeed irrespective of the mode of delivery. Interestingly, the women, who delivered vaginally, had mean BMI of 24

\footnotetext{
${ }^{1}$ http://cgenome.net/calypso/

${ }^{2}$ http://huttenhower.sph.harvard.edu/galaxy/
} 
(SD \pm 2.6 ), except the Chinese group with mean BMI of 21.9 $(\mathrm{SD} \pm 1.5)$. South African and Finnish women, who delivered by cesarean section had mean BMI of 26. All women, who delivered via C-section, were given prophylactic antibiotics, except Finnish women where no prophylaxis is routinely used as per the hospital policy. All South African women got cefazolin before C-section whereas Spanish women either got cefazolins or penicillin related. Chinese women who delivered by $\mathrm{C}$-section either got metronidazole, cephalosporins or azithromycin.

\section{Breast Milk Fatty Acid Profile Based on Phospholipids and Triacylglycerols}

Our results showed that the fatty acid profile of phospholipids varied across the four countries with significant differences in the levels of total PUFA ( $p<0.001)$, n-6 PUFA ( $p<0.001)$, n-3 PUFA $(p=0.003)$, and SAFA $(p<0.001)$. Interestingly, north and south of Europe, i.e., Spain and Finland differed significantly in the n-3 $(p<0.001)$ and $n-6(p=0.007)$ fatty acid levels in TAG.

In China, the levels of SAFAs $(34.7 \%$ of total TAG) in TAG were lower $(p<0.001)$ than in the other areas, with relative order corresponding to (Finland $>$ South Africa $>$ Spain)
(Supplementary Table 1). The Chinese and Finnish samples had relatively higher n-3 PUFA than the Spanish and South African samples. On the other hand, n-6 PUFA was found to be highest $(34.84 \%$ in $\mathrm{PL}, 25.66 \%$ in TAG) in Chinese samples (Figures 1A,B). These differences observed in TAG and phospholipids in the Chinese samples were also revealed by PCA, which showed distinct Chinese clustering compared to the samples from other areas (Figures 1C,D).

The impact of the mode of delivery is not consistent across the countries. However, fatty acid profile differed between the countries as the South African women who delivered by cesarean section had higher MUFA $(p<0.001)$ in their milk than those women who delivered by the vaginal delivery; whereas Spanish women who delivered by vaginal delivery had higher MUFA in TAG $(p=0.003)$ (Table $\mathbf{1})$.

\section{Microbiota Compositional Analysis}

Overall, the sequencing resulted in 911,547 clean and filtered sequences with mean count of $11,538( \pm 9,025 \mathrm{SD})$ reads per sample. Milk microbiota composition differed significantly across all the countries (ANOSIM test, $p=0.002$ ). However, Finnish and South African samples had relatively similar profiles for
A

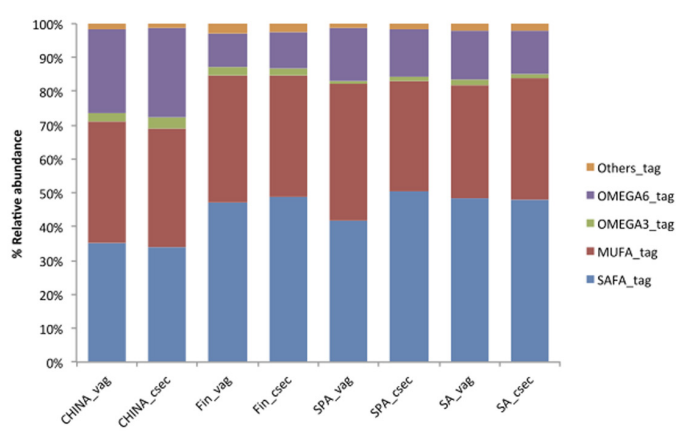

C

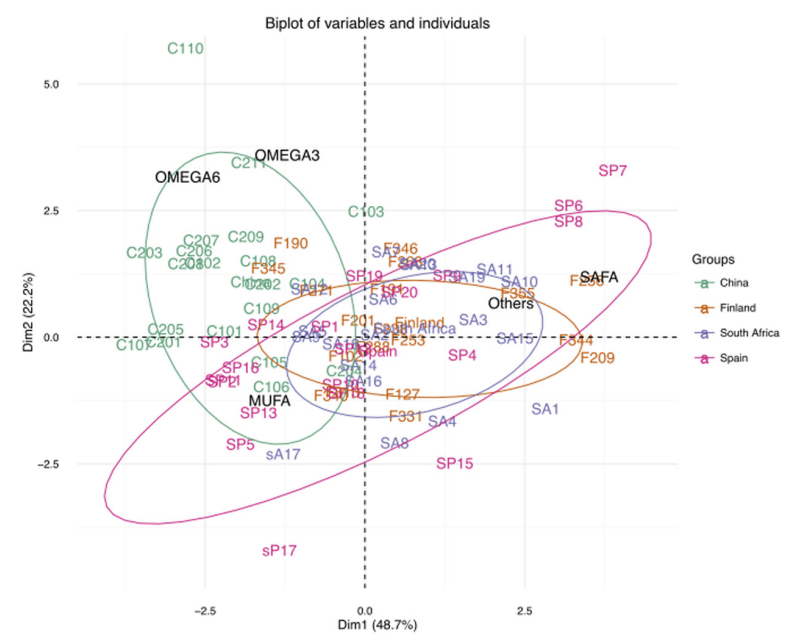

B

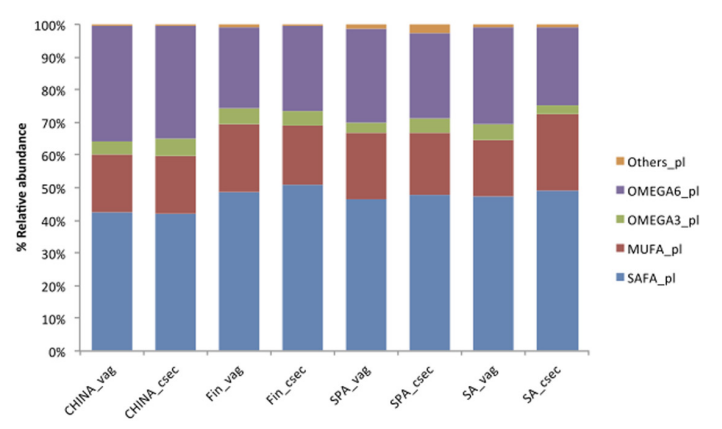

D

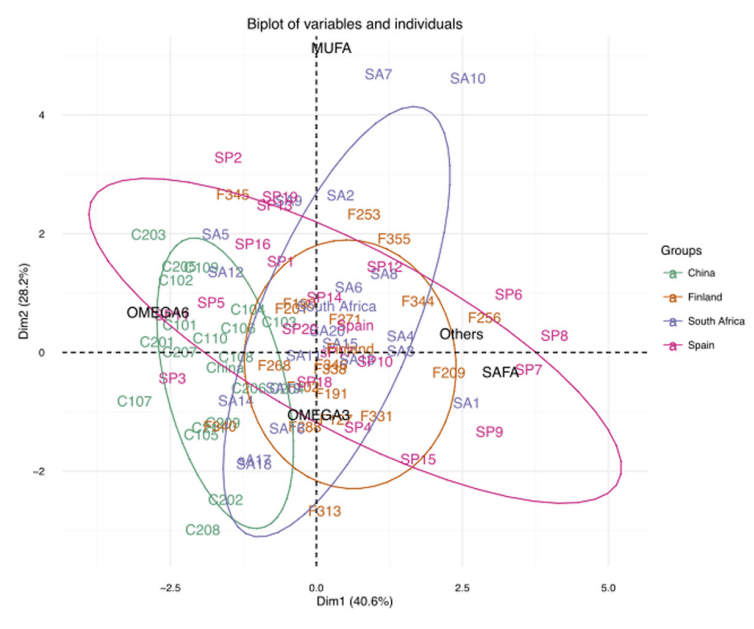

FIGURE 1 | Comparison of percentage relative abundances of fatty acids taking mode of delivery and country as factors (A) Triacyglycerols (B) Phospholipids; (C) PCA of triacyglycerols (D) PCA of phospholipids, ellipses in the plot represent $95 \%$ confidence interval of grouping based on countries. 


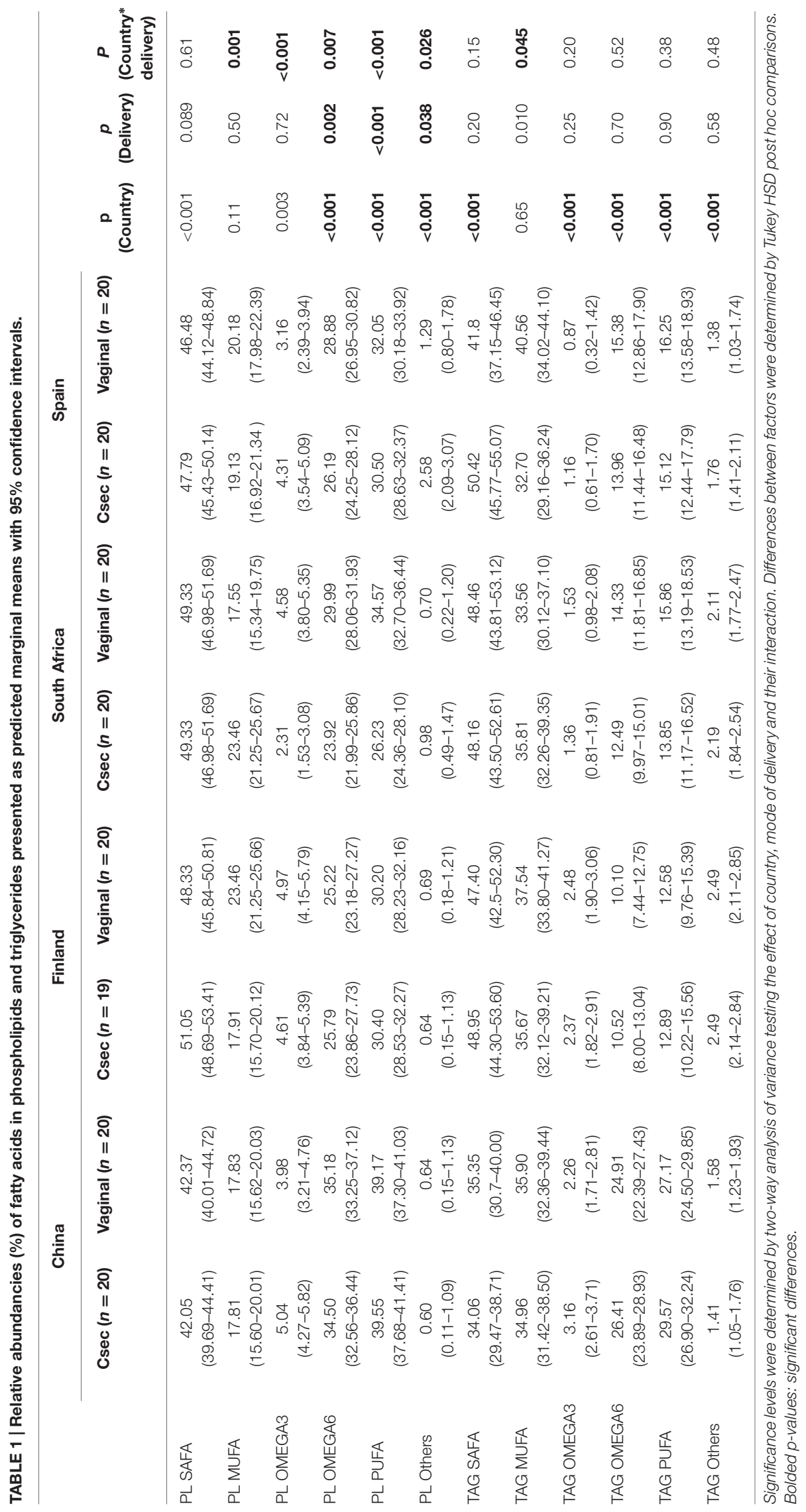


vaginal and cesarean delivery (Figure 2A). Spanish women who delivered vaginally had the highest level of Bacteroidetes in breast milk in comparison to women from other countries. In general, South African women had significantly higher relative abundance of Proteobacteria $(p=0.004)$ when compared to other countries. This could have been attributed to higher level of Enterobacteriaceae and Pseudomonadaceae family observed in the milk samples of the South African and Spanish women $(p<0.05)$. The samples from Finland had higher levels of Firmicutes and lower levels of Proteobacteria when compared to the samples from other countries $(p=0.004)$. Among the mothers with vaginal delivery, the Chinese women had the highest level of Actinobacteria. At genus level, Chinese women had higher levels of Streptococcus, and Spanish women higher levels of Propionibacterium and Pseudomonas in Spanish when compared to women from all countries with mode of delivery as factor $(p<0.05)$ (Figure 2B).

Spanish mothers who delivered by cesarean section had decreased alpha-diversity $(p=0.013)$ and hallmarked by higher level of Proteobacteria in comparison to all women from other countries. Overall, other diversity indices across all the study regions were similar. Within the Spanish group, women who delivered vaginally had significantly higher diversity $(p=0.021)$ when compared to those who delivered by C-section.

Overall microbial community at OTU level was significantly different in all countries suggesting a geographical areadependent microbiota. The European areas (Spain and Finland) had significant differences in microbial communities even when mode of delivery was taken into account $(p<0.05)$. RDA analysis at OTU level showed that the microbiota profile of Spanish and South African milk samples were more diverse when compared to those of Finnish and Chinese women who formed tight clusters (Figure 3B). Due to the impact of individual variability and geographical area, we identified a core microbiome (at family level) with 23 phylotypes that constituted the core milk microbiome across all the countries (Figure 3A). Lactobacillaceae was found uniquely in samples from Finland, Bifidobacteriaceae found only in South African women and Enterococcaceae in samples from all the countries except China (Supplementary Table 2).

\section{Imputed Metagenomic Analysis and Metabolic Pathway Prediction}

Metagenomic prediction based on PICRUSt analysis and LEfSe results revealed siginificant difference in abundance of KEGG pathways in South African, Spanish and Chinese women at L2 of KEGG pathway (Figure 4). South African samples were differentially mapped to metabolic pathways as predicted by KEGG based on annotation at L2 and L3 levels (Figure 4). Some of the major differential pathways included fat digestion and absorption, biotin metabolism and histidine metabolism. Spain and South African women had significantly higher while Finnish women had significantly lower number of reads mapped to lipid metabolism, amino acid metabolism and carbohydrate metabolism when compared among all the countries $(p<0.05)$.

\section{Association of BMI, Fatty Acid Profile with Microbiota Composition}

Redundancy analysis at OTU level, taking all the fatty acid as factors, revealed that geographical location $(p=0.003)$ is one of major contributing factor associated with differences in microbiota observed in all women. Moreover, the fatty acids, SAFA of TAG $(p=0.002)$ and PUFA of phospholipids $(p=0.05)$ also found to be contributing factor associated with the differences observed in microbiota.

Regression analysis at phyla level, taking BMI as a factor, showed that in more than $98 \%$ of women (irrespective of their country) Firmicutes was positively associated with BMI. In all samples, MUFA in TAG were found to be negatively associated with Proteobacteria $(r=-0.43, p<0.05)$. In more than $90 \%$ of participating women, MUFA and PUFA in TAG were found to have a significant positive association while SAFA in triacylglycerols was negatively associated, with the Streptococcus and Acinetobacter genus. SAFA (in both TAG and phospholipids) was also found to be positively associated with Pseudomonas. In phospholipids, Lactobacillus genus was negatively associated with MUFA $(r=-0.23, p=0.04)$ and $n-3$ PUFA was negatively associated with Bifidobacterium genus $(r=-0.26, p=0.03)$. The heat map generated from Pearson correlation analysis based on microbiota at genus level with major categories of TAG and phospholipids showed that higher abundance of Pseudomonas was correlated with lower levels of MUFA in TAG, whereas higher abundance of Streptococcus genus were correlated with lower levels of SAFA in TAG. Within TAG, Staphylococcus genus was found to be negatively correlated with MUFA (Figure 5).

\section{DISCUSSION}

Breastfeeding is one of the most important practices, which is recommended worldwide for the adequate nutrition and health of newborns. We reported the effect of environment (geographical area and/or lifestyle) in the milk microbiota composition, lipids and the relationship between them. These factors are transferred to infant during breast-feeding and will likely create a hallmark in infant gut microbiome and immune system maturation. All the milk samples were obtained with the same protocol (to avoid bias) and processed in Finland. Sequencing was carried out at South Africa, to avoid effect of different batches (if processed at their respective locations).

Breast milk fat is a major source of energy, providing over $40 \%$ of the total energy for infants during first 6 months of life. Fat globules in breast milk consist of TAG (98-99\% of milk lipids) and small amount of monoacylglycerols, diacylglycerols, free fatty acids surrounded by milk fat membrane of different phospholipids (Delplanque et al., 2015). Some of the indispensable lipid components include PUFAs and long chain PUFAs (LC-PUFA) of the n-6 and n-3 series and lipid soluble vitamins. A recent study reported complex interactions between fatty acids and protein profiles which were influenced by lactation stage and also, 


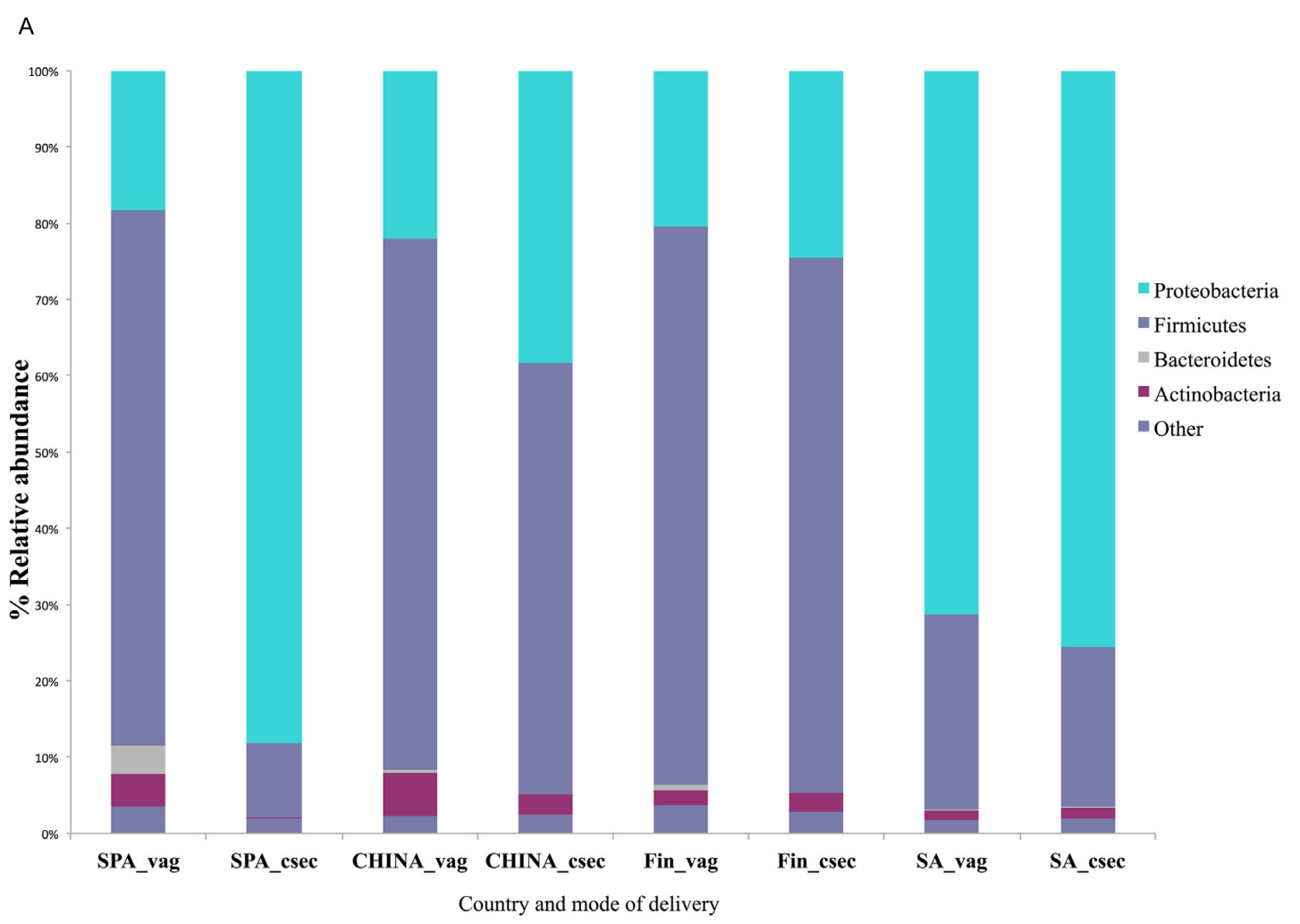

B

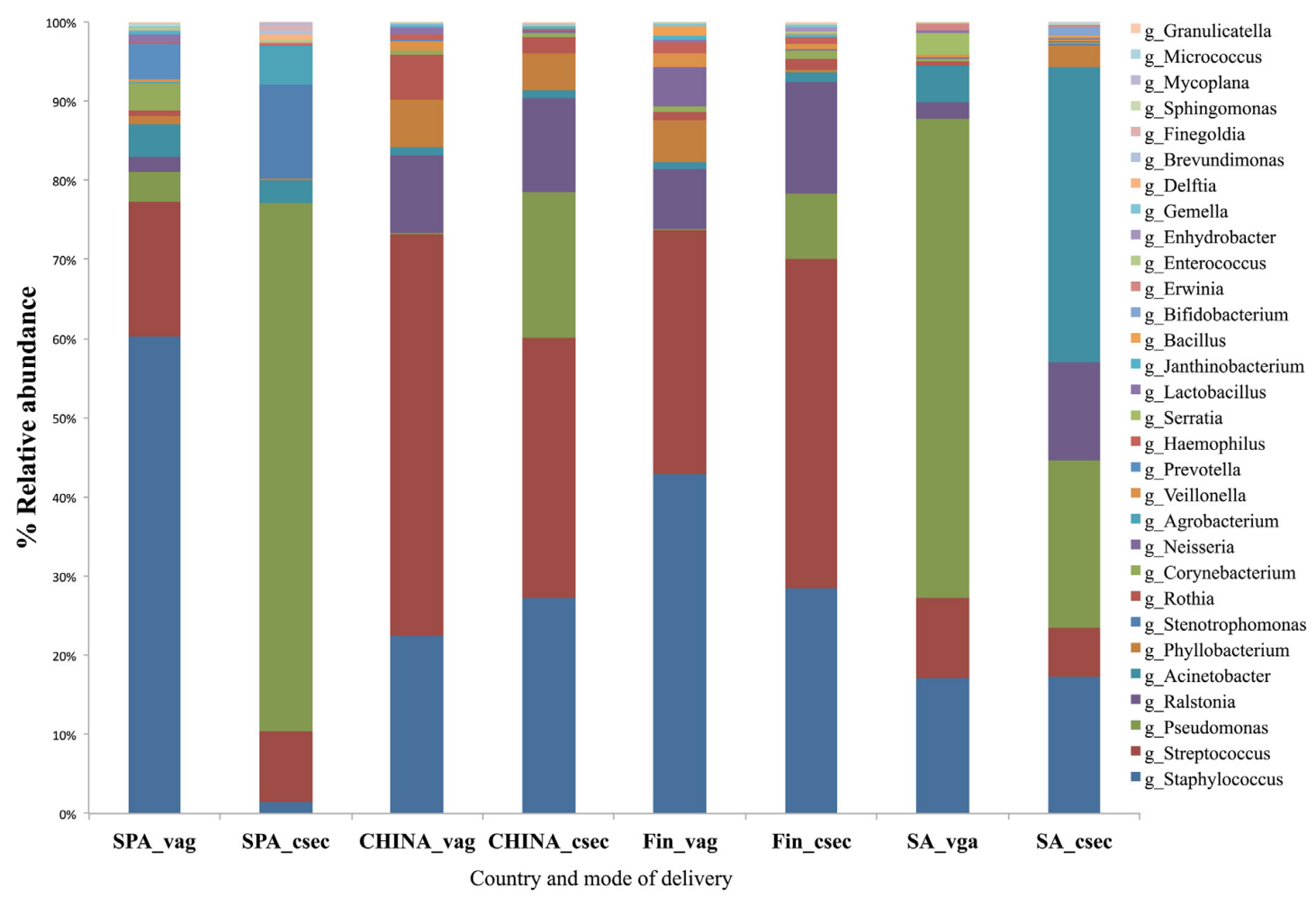

FIGURE 2 | Comparison of percentage relative abundances taking mode of delivery and country as factor at (A) Phyla level (top 5 phylotypes based on relative abundance), (B) Genus level (top 30 genera based on total relative abundance). 
A

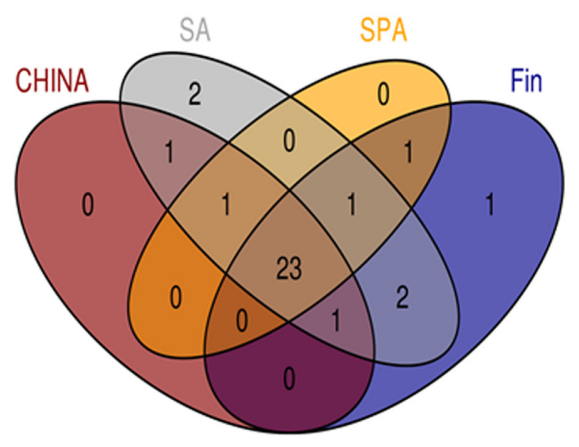

B

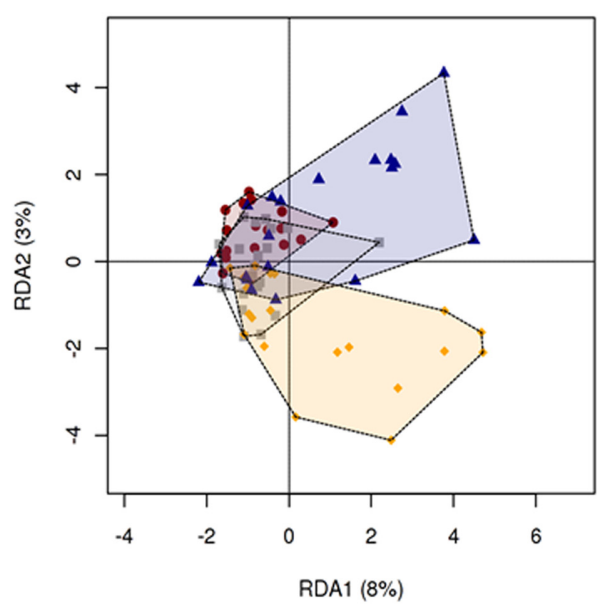

RDA OTU

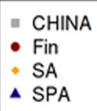

FIGURE 3 | (A) Shared phylotypes at family level across countries. (B) RDA analysis at OTU level, across different countries.

A

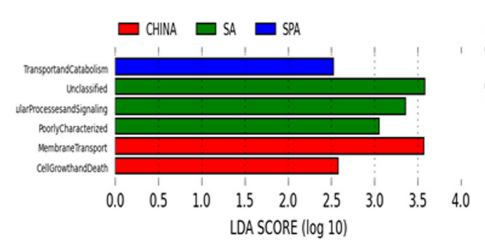

B

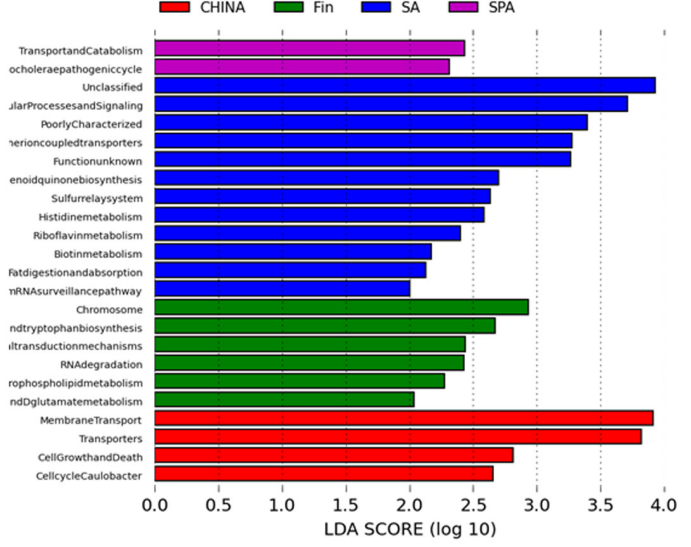

FIGURE 4 | PICRUSt based predicted metagenome using KEGG annotation at levels (A) L2 (B) L3, representing LDA scores for differentially abundant genes/pathways (Log LDA $=2$ ) across different countries.

gestational age (Collado et al., 2015). Our results demonstrated differences in human milk fat composition and fatty acid profiles of study milks between the four countries. The highest variability was observed in n-6 PUFA in TAG fatty acids and in phospholipids. In general, human milk fat content exhibits high degree of inter-individual variability, with maternal nutrition, adiposity and health being some of the important factors. This is also confirmed by two independent studies, in a Danish study which involved 244 women revealed that fat content varied from $1.8 \mathrm{~g} / \mathrm{dl}$ at lower 2.5 percentile to $8.9 \mathrm{~g} / \mathrm{dl}$ at the upper 97.5 percentile, and in another study based on women from United States the range varied from $0.7 \mathrm{~g} / \mathrm{dl}$ to $7.06 \mathrm{~g} / \mathrm{dl}$ (Robinson and Caplan, 2015). The variations observed in Chinese population, could be attributed to number of factors majorly to maternal nutrition.
Recent studies have reported the high diversity of microbial composition in human milk (Hunt et al., 2011; CabreraRubio et al., 2012, 2016; Fernandez et al., 2013; Jost et al., 2013). We also confirmed previous findings as those reported in the Finnish population (Cabrera-Rubio et al., 2012) and Spanish population (Cabrera-Rubio et al., 2016), that mode of delivery associates with milk microbiome but the impact differed depending on the country. In addition to the differences observed in milk microbiome, another recent report in USA has shown differences in glycolysation pattern based on mode of delivery (Hoashi et al., 2015). However, another study on milk microbiota of 39 Canadian women could not find any difference based on mode of birth, which could be also because of high number $(n=28)$ of preterm deliveries (Urbaniak et al., 2016). It is also well-established that genetics and environmental factors influence gut microbiota composition. Big 


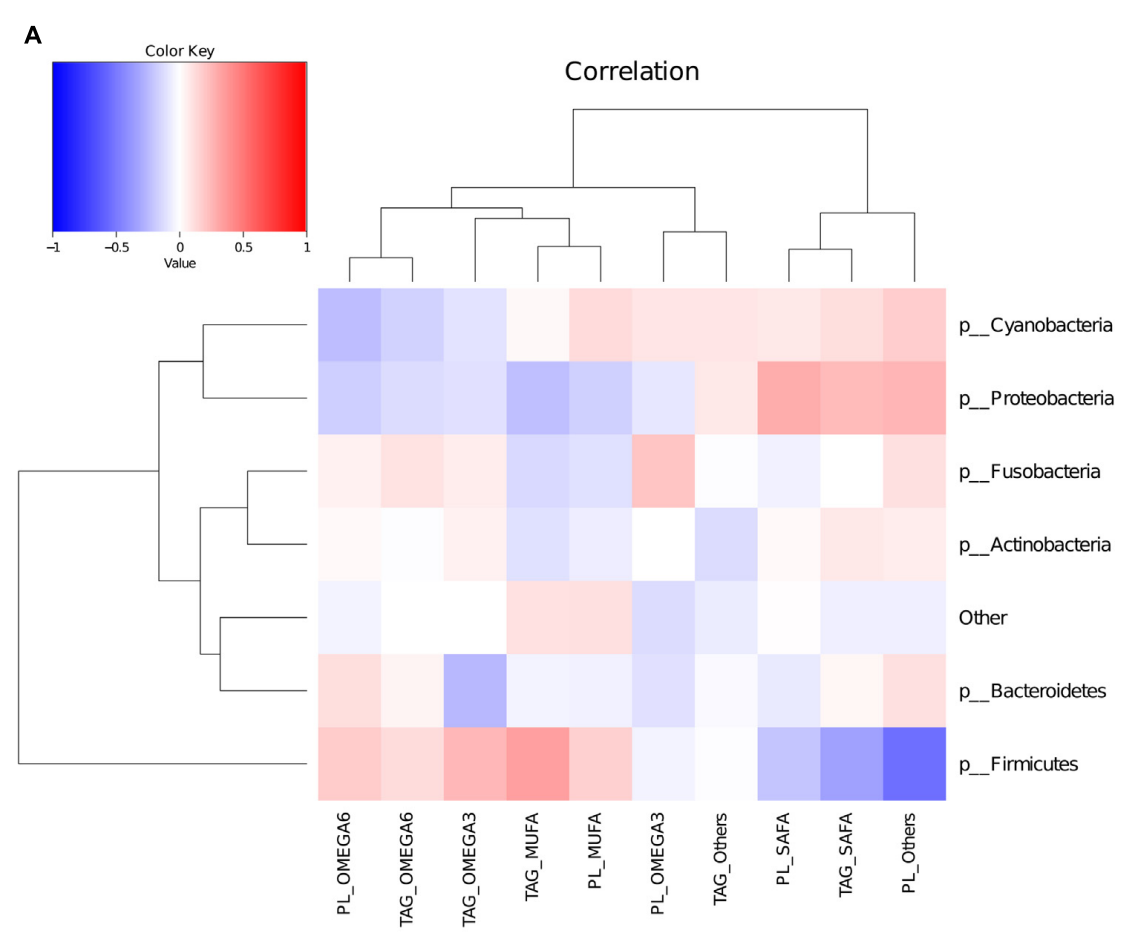

B
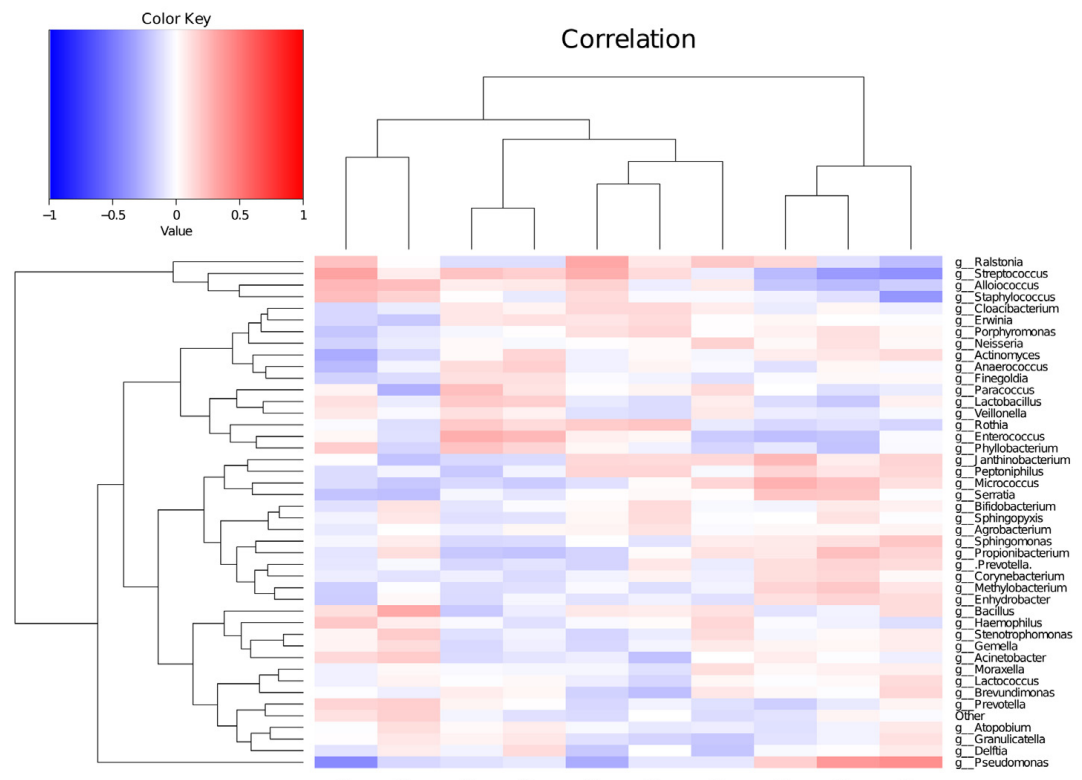

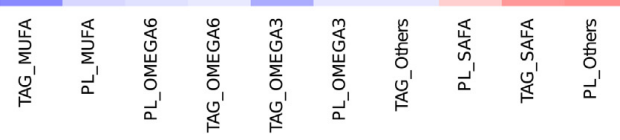

FIGURE 5 | Heatmap to show correlation between lipidomic data and microbiota composition at phylum level (A) and genus level (B).

worldwide cohorts - MetaHIT (European), HMP (American), and Chinese diabetes cohorts-reported that the inter-country variation in gut microbiota composition significantly exceeded inter-personal variation (Lloyd-Price et al., 2016). A study targeting gut microbiota from five European countries (Sweden, United Kingdom, Germany, Italy, and Spain), representing varied life style characteristics, showed that higher proportion of bifidobacteria was found in Northern European countries 
while Bacteroides was more prominent in southern countries (Fallani et al., 2010). Interestingly, we also found in this study that the breast milk samples from Spanish women exhibited higher diversity than those from northern Europe; although our study is focused on breast milk which may in turn affect the infant gut microbiota. Despite the great inter-individual variability, accumulating data show that breast milk microbiota is dominated by Staphylococci, Streptococci, specific Proteobacteria group, and Propionibacteria (Hunt et al., 2011). Additionaly, specific anaerobic bacterial groups such as Bifidobacterium, Bacteroides, Parabacteroides and members of Clostridia have been also detected as common constituents of breast milk (Sinkiewicz and Ljunggren, 2008; Ted et al., 2014).

Overall, metagenomic prediction revealed that some of the genes associated with lipid, amino acid, and carbohydrate metabolism were predominated in South African and Spanish women, which was also observed in overall microbial community similarity. These variations in pathways could also be due to differences in major metabolites present in milk and their interaction with microbes. However, transcriptome profile of human breast milk also suggested that the dietary modifications due to fat intake was not reflected in expression of genes associated with fatty acid uptake/synthesis or cell-cycle regulation (Yahvah et al., 2015). Microbiota and fatty acid compositional analysis revealed that the geographic origin is a more important factor for the observed differences in women, although there were differences based on mode of delivery, this observation is in concordance with earlier reported (Fallani et al., 2010).

When combining fatty acid profile and milk microbiota, we found specific association of fatty acid with microbial groups. Human milk fatty acids may influence the composition and activity of specific bacteria as demonstrated here. Fat intake is rapidly reflected in human milk fatty acid composition, which in turn may favor specific bacteria utilizing certain fatty acids incorporating them into their cell walls. Changes in the fatty acid profiles in microbial cell surfaces may advance or hinder their ability to colonize the infant gut. Our study suggests that Staphylococcus genus and other bacterial genus as Pseudomonas were negatively correlated with MUFA in TAG, whereas Streptococcus genus was negatively correlated with SAFA in TAG. In phospholipids, the levels of Bifidobacterium and Lactobacillus genera showed a negative association with MUFA and n-3 PUFA may suggest specific interactions, which need further validation. A previous study comparing gut microbiota profile of lactating women, has shown specific negative association of $\mathrm{n}-3$ fatty acids with Bacteroidetes phyla and positive association with Firmicutes phyla (Carrothers et al., 2015). We could not find similar associations in this study, which could have been attributed to difference in sampling sites as our study is focused on breast milk and used geographical location as one of the major contributing factor. Also, the number of samples in each group could be one of the limiting factors in finding strong association with specific microbial groups. Regional dietary difference may also contribute affects the breast milk composition, for example due to increase consumption of n-3 PUFA (linoleic acid) containing vegetable oil products in western diet, has led to increase in linoleic acid concentration from 50 to $90 \%$ of the PUFAs (Liu et al., 2016). In another study involving 514 health lactating chinese mothers did not find correlation of dietary intake of Linoleic acid with milk higher dihomo $\gamma$-linolenic acid, arachidonic acid or docosatetraenoic level, which they proposed could be due to gene polymorphism affecting fat storage (Liu et al., 2016). SFA present in breast milk is related with carbohydrate and fats in daily diets as well as total energy intake and mobilization of adipose tissue (Krešić et al., 2013). The fatty acid composition along with specific microbial constitution might reflect directly in immune programming in infants. Bifidobacterium, Lactobacillus, Bacteroides, and Clostridium spp. have been associated with immunological functions such tolerance, mucus production, tight junction expression and $\mathrm{T}$ helper cell balance, which helps in intestinal barrier homeostasis (Walker and Iyengar, 2015). Also dietary intakes have shown to have strong impact on gut microbiota. Such associations should be further characterized to understand the role of mother's nutrition and fatty acid intake on breast milk fatty acid and microbiota profiles. There is also evidence that human milk hormones are directly associated with gut microbial functional pathways implicated in infants intestinal inflammation (Lemas et al., 2016). Recent study based on early gut microbiota of infants from Finland, Estonia and Russia has shown that differences in Bacteroides spp. derived LPS when compared to Escherichia coli derived LPS has differential immunogenic roles, which could be associated with higher incidences of auto-immune diseases prevalent in Northern Europe (Vatanen et al., 2016). Such studies including ours provide new insights and may propose novel tools for dietary recommendations and nutrition counseling for breast-feeding mothers.

\section{CONCLUSION}

Our results demonstrate differences in the composition of lipids and microbiota in breast milk in different geographic regions and offer a new insight to the differences in development of gut microbiota in infants in different geographic areas. The impact of mode of delivery on milk microbiota composition appears to be even more important within all geographical regions. Furthermore, our study shows distinct association between fatty acid composition and individual microbiota in breast milk, suggesting important role of lipids in shaping the microbial profile which likely one important factor influencing the infant gut microbiota development.

\section{AUTHOR CONTRIBUTIONS}

The work was initially designed by HK, ET, MC, KL, BY, EI, and SS. Data contributors were HK, ET, AK, JA, YZ, and MN. The manuscript was drafted by HK, MC, ET, KL, and SS edited and approved by all authors. 


\section{FUNDING}

The study was funded by the strategy funding programme of the University of Turku "Finnish-Chinese Cooperation in Food Quality and Safety" (Decision number: 951/210/2014). Sequencing carried out at CPGR, South Africa, thanks to funding from the National Institutes of Allergy and Infectious Disease 1U01AI110466-01A1, H3Africa Initiative. Chinese group acknowledges the support from Key Projects of Beijing Science \& Technology (D141100004814002), and Natural scientific foundation of Beijing (Z140001). MCC (Spain) would like to gratefully acknowledge European Research Council ERC Starting Grant 639226-MAMI.

\section{REFERENCES}

Aaltonen, J., Ojala, T., Laitinen, K., Poussa, T., Ozanne, S., and Isolauri, E. (2011). Impact of maternal diet during pregnancy and breastfeeding on infant metabolic programming: a prospective randomized controlled study. Eur. J. Clin. Nutr. 65, 10-19. doi: 10.1038/ejcn.2010.225

Boersma, E. R., Offringa, P. J., Muskiet, F., Chase, W. M., and Simmons, I. J. (1991). Vitamin E, lipid fractions, and fatty acid composition of colostrum, transitional milk, and mature milk: an international comparative study. Am. J. Clin. Nutr. $53,1197-1204$

Brenna, J. T., Varamini, B., Jensen, R. G., Diersen-Schade, D. A., Boettcher J. A., and Arterburn, L. M. (2007). Docosahexaenoic and arachidonic acid concentrations in human breast milk worldwide. Am. J. Clin. Nutr. 85, $1457-$ 1464.

Cabrera-Rubio, R., Collado, M. C., Laitinen, K., Salminen, S., Isolauri, E., and Mira, A. (2012). The human milk microbiome changes over lactation and is shaped by maternal weight and mode of delivery. Am. J. Clin. Nutr. 96, 544-551. doi: 10.3945/ajcn.112.037382

Cabrera-Rubio, R., Mira-Pascual, L., Mira, A., and Collado, M. C. (2016). Impact of mode of delivery on the milk microbiota composition of healthy women. J. Dev. Orig. Health Dis. 7, 54-60. doi: 10.1017/s2040174415001397

Caporaso, J. G., Kuczynski, J., Stombaugh, J., Bittinger, K., Bushman, F. D., Costello, E. K., et al. (2010). QIIME allows analysis of highthroughput community sequencing data. Nat. Methods 7, 335-336. doi: 10.1038/nmeth.f.303

Caporaso, J. G., Lauber, C. L., Walters, W. A., Berg-Lyons, D., Huntley, J., Fierer, N., et al. (2012). Ultra-high-throughput microbial community analysis on the Illumina HiSeq and MiSeq platforms. ISME J. 6, 1621-1624. doi: 10.1038/ismej.2012.8

Carrothers, J. M., York, M. A., Brooker, S. L., Lackey, K. A., Williams, J. E., Shafii, B., et al. (2015). Fecal microbial community structure is stable over time and related to variation in macronutrient and micronutrient intakes in lactating women. J. Nutr. 145, 2379-2388. doi: 10.3945/jn.115.211110

Christie, W. W. (1982). A simple procedure for rapid transmethylation of glycerolipids and cholesteryl esters. J. Lipid Res. 23, 1072-1075.

Collado, M. C., Laitinen, K., Salminen, S., and Isolauri, E. (2012). Maternal weight and excessive weight gain during pregnancy modify the immunomodulatory potential of breast milk. Pediatr. Res. 72, 77-85. doi: 10.1038/pr. 2012.42

Collado, M. C., Santaella, M., Mira-Pascual, L., Martinez-Arias, E., KhodayarPardo, P., Ros, G., et al. (2015). Longitudinal study of cytokine expression, lipid profile and neuronal growth factors in human breast milk from term and preterm deliveries. Nutrients 7, 8577-8591. doi: 10.3390/nu7105415

Delplanque, B., Gibson, R., Koletzko, B., Lapillonne, A., and Strandvik, B. (2015). Lipid quality in infant nutrition: current knowledge and future opportunities. J. Pediatr. Gastroenterol. Nutr. 61, 8-17. doi: 10.1097/MPG.0000000000000818

Fallani, M., Young, D., Scott, J., Norin, E., Amarri, S., Adam, R., et al. (2010). Intestinal microbiota of 6-week-old infants across Europe: geographic influence beyond delivery mode, breast-feeding, and antibiotics. J. Pediatr. Gastroenterol. Nutr. 51, 77-84. doi: 10.1097/MPG.0b013e3181d1b11e

\section{ACKNOWLEDGMENTS}

We acknowledge the support staff and technicians for the help in sample collection and storage. Heta Haikonen is thanked for technical assistance in the lipid analysis.

\section{SUPPLEMENTARY MATERIAL}

The Supplementary Material for this article can be found online at: http://journal.frontiersin.org/article/10.3389/fmicb. 2016.01619

Fernandez, L., Langa, S., Martin, V., Jimenez, E., Martin, R., and Rodriguez, J. M. (2013). The microbiota of human milk in healthy women. Cell Mol. Biol. (Noisy-le-grand) 59, 31-42.

Folch, J., Lees, M., and Sloane-Stanley, G. (1957). A simple method for the isolation and purification of total lipids from animal tissues. J. Biol. Chem. 226, 497-509.

Hamilton, J. G., and Comai, K. (1988). Rapid separation of neutral lipids, free fatty acids and polar lipids using prepacked silica Sep-Pak columns. Lipids 23, 1146-1149. doi: 10.1007/BF02535281

Hoashi, M., Meche, L., Mahal, L. K., Bakacs, E., Nardella, D., Naftolin, F., et al. (2015). Human milk bacterial and glycosylation patterns differ by delivery mode. Reprod. Sci. 23, 902-907. doi: 10.1177/1933719115623645

Hunt, K. M., Foster, J. A., Forney, L. J., Schütte, U. M., Beck, D. L., Abdo, Z., et al. (2011). Characterization of the diversity and temporal stability of bacterial communities in human milk. PLOS ONE 6:e21313. doi: 10.1371/journal.pone.0021313

Jost, T., Lacroix, C., Braegger, C. P., Rochat, F., and Chassard, C. (2013). Vertical mother-neonate transfer of maternal gut bacteria via breastfeeding. Environ. Microbiol. 16, 2891-2904. doi: 10.1111/1462-2920.12238

Kelishadi, R., and Farajian, S. (2014). The protective effects of breastfeeding on chronic non-communicable diseases in adulthood: a review of evidence. Adv. Biomed. Res. 3, 3. doi: 10.4103/2277-9175.124629

Khodayar-Pardo, P., Mira-Pascual, L., Collado, M. C., and Martinez-Costa, C. (2014). Impact of lactation stage, gestational age and mode of delivery on breast milk microbiota. J. Perinatol. 34, 599-605. doi: 10.1038/jp.2014.47

Krešić, G., Dujmović, M., Mandić, M. L., and Delaš, I. (2013). Relationship between Mediterranean diet and breast milk fatty acid profile: a study in breastfeeding women in Croatia. Dairy Sci. Technol. 93, 287-301. doi: 10.1007/s13594-0130125-6

Langille, M. G., Zaneveld, J., Caporaso, J. G., McDonald, D., Knights, D., Reyes, J. A., et al. (2013). Predictive functional profiling of microbial communities using 16S rRNA marker gene sequences. Nat. Biotechnol. 31, 814-821. doi: $10.1038 /$ nbt.2676

Lemas, D. J., Young, B. E., Baker, P. R. II, Tomczik, A. C., Soderborg, T. K., Hernandez, T. L., et al. (2016). Alterations in human milk leptin and insulin are associated with early changes in the infant intestinal microbiome. Am. J. Clin. Nutr. 103, 1291-1300. doi: 10.3945/ajcn.115.126375

Liu, G., Ding, Z., Li, X., Chen, X., Wu, Y., and Xie, L. (2016). Relationship between polyunsaturated fatty acid levels in maternal diets and human milk in the first month post-partum. J. Hum. Nutr. Diet. 29, 405-410. doi: 10.1111/jhn.12337

Lloyd-Price, J., Abu-Ali, G., and Huttenhower, C. (2016). The healthy human microbiome. Genome Med. 8, 51. doi: 10.1186/s13073-016-0307-y

Mäkelä, J., Linderborg, K., Niinikoski, H., Yang, B., and Lagstrom, H. (2013). Breast milk fatty acid composition differs between overweight and normal weight women: the STEPS Study. Eur. J. Nutr. 52, 727-735. doi: 10.1007/s00394-0120378-5

R Core Team (2010). R: A Language and Environment for Statistical Computing. Vienna: R Foundation for Statistical Computing.

Robinson, D. T., and Caplan, M. S. (2015). Linking fat intake, the intestinal microbiome, and necrotizing enterocolitis in premature infants. Pediatr. Res. 77, 121-126. doi: 10.1038/pr.2014.155 
Schmieder, R., and Edwards, R. (2011). Quality control and preprocessing of metagenomic datasets. Bioinformatics 27, 863-864. doi: 10.1093/bioinformatics/btr026

Sinkiewicz, G., and Ljunggren, L. (2008). Occurrence of Lactobacillus reuteri in human breast milk. Microb. Ecol. Health Dis. 20, 122-126. doi: 10.1080/08910600802341007

Soto, A., Martín, V., Jiménez, E., Mader, I., Rodríguez, J. M., and Fernández, L. (2014). Lactobacilli and bifidobacteria in human breast milk: influence of antibiotherapy and other host and clinical factors. J. Pediatr. Gastroenterol. Nutr. 59, 78-88. doi: 10.1097/MPG.0000000000000347

Ted, J., Christophe, L., Christian, P. B., Florence, R., and Christophe, C. (2014). Vertical mother-neonate transfer of maternal gut bacteria via breastfeeding. Environ. Microbiol. 16, 2891-2904. doi: 10.1111/1462-2920.12238

Urbaniak, C., Angelini, M., Gloor, G. B., and Reid, G. (2016). Human milk microbiota profiles in relation to birthing method, gestation and infant gender. Microbiome 4, 1-9. doi: 10.1186/s40168-015-0145-y

Vatanen, T., Kostic, A. D., d'Hennezel, E., Siljander, H., Franzosa, E. A., Yassour, M., et al. (2016). Variation in microbiome LPS immunogenicity contributes to autoimmunity in humans. Cell 165, 842-853. doi: 10.1016/j.cell.2016.04.007
Walker, W. A., and Iyengar, R. S. (2015). Breast milk, microbiota and intestinal immune homeostasis. Pediatr. Res. 77, 220-228. doi: 10.1038/pr.2014.160

Yahvah, K. M., Brooker, S. L., Williams, J. E., Settles, M., McGuire, M. A., and McGuire, M. K. (2015). Elevated dairy fat intake in lactating women alters milk lipid and fatty acids without detectible changes in expression of genes related to lipid uptake or synthesis. Nutr. Res. 35, 221-228. doi: 10.1016/j.nutres.2015.01.004

Conflict of Interest Statement: The authors declare that the research was conducted in the absence of any commercial or financial relationships that could be construed as a potential conflict of interest.

Copyright (c) 2016 Kumar, du Toit, Kulkarni, Aakko, Linderborg, Zhang, Nicol, Isolauri, Yang, Collado and Salminen. This is an open-access article distributed under the terms of the Creative Commons Attribution License (CC BY). The use, distribution or reproduction in other forums is permitted, provided the original author(s) or licensor are credited and that the original publication in this journal is cited, in accordance with accepted academic practice. No use, distribution or reproduction is permitted which does not comply with these terms. 